\title{
Program Amazon without Fire Brazil: contribution of international cooperation to national strategies and policies
}

\author{
Lara Steil ${ }^{1 *}$, Roberto Bianchi ${ }^{2}$, André Luiz Pereira Galvão ${ }^{3}$
}

\author{
${ }^{1}$ Brazilian Institut for Environment and Renewable Natural Resources (Ibama), Brasília, Brazil \\ ${ }^{2}$ Coordinator of the program in Brazil and Bolivia by the Italian side \\ ${ }^{3}$ Brazilian Cooperation Agency / Ministry of External Relations (MRE), Brasilia, Brazil \\ *Contact email: lara.steil@ibama.gov.br
}

\begin{abstract}
The Program Amazon without Fire Brazil (PASF-Br) was an initiative of the Brazilian government in partnership with the Italian government carried out from 1999 to 2008. Taking into account the many fire-prone areas spread along the arc of deforestation, the program aimed to reduce the incidence of wildfires in the Brazilian Amazon and to improve the living conditions of rural communities. Government agencies, civil Society organizations and rural communities from 34 municipalities in the states of Acre, Mato Grosso and Para took part f PASF-Br. The strategies adopted included: a) development of Municipal/Community Fire Prevention Protocols; b) implementation of demonstration units (DU); c) environmental education; d) training on fire prevention and fighting; e) capacity building by addressing the training of multipliers; f) implementation of action by the multipliers. The results showed an increase in the aware and engagement of local communities related to the reduction of fire-use for rural purposes, decreasing the wildfires locally. The families that implemented the DU had an increase in family income. This outcome together with the multipliers' performance promoted the expansion of the program's area of operation, leading rural community members initially not involved in the program, to seek the adoption of the program's strategies. PASF-Br influenced national strategies and policies. The National Center for Prevention and Fighting Wildfires - Prevfogo adopted the strategies of the program. In addition, the new forest code of Brazil, in its article 40, establishes that the replacement of fire-use in rural areas should be considered. The successful outcomes of the program in Brazil lead to Bolivia and Ecuador to establish trilateral cooperations with Brazil and Italy to develop the same program. The Pasf-Bolivia was completed in 2017 and the PasfEcuador is in progress. Clearly, this program has identified that international cooperation is an important mechanism for improving national strategies. The actions proposed by the program are in line with the scope of the integrated fire management approach, as they provide a broader dialogue between the various stakeholders and propose the replacement of fire-use in an ecosystem, the Amazon, not adapted to this natural element.
\end{abstract}

Keywords: Amazon without Fire, alternative techniques to fire-use, demonstration units. 